\title{
Nasal and ophthalmomyiasis: case report
}

\author{
I SMillie, P K S GubBi, H C Cocks
}

\begin{abstract}
Objective: We report a rare case of a 51-year-old woman with ocular and nasal infestation by Oestrus ovis.

Method: Clinical case report and review of current literature regarding nasal and ophthalmomyiasis.

Results: Myiasis is infestation of the tissues and organs of vertebrates by certain dipteran fly larvae. Oestrus ovis myiasis is endemic in the region from North Africa to South Asia, but few cases are reported within the UK. A 51-year-old patient presented and was treated successfully in Sunderland Royal Hospital for combined ocular and nasal infestation with Oestrus ovis.

Conclusions: Oestrus ovis has a largely subtropical distribution; however, it is important to remember the diversity of disease which can present within the UK from an external source. This point is illustrated by this rare case, which highlights the fact that prompt treatment can avoid poor outcomes for our patients.
\end{abstract}

Key words: Myiasis; Nose; Ophthalmomyiasis; Oestrus Ovis

\section{Introduction}

Myiasis is a parasitic disease caused by the larvae of numerous dipteran fly species, including the sheep bot fly Oestrus ovis. This species is an obligate parasite in the nasal cavities and frontal sinuses of sheep, but may also cause infestation in humans. ${ }^{1}$ The primary site of infestation is usually the nose, ears, eyes and surrounding skin, but can also include the pharynx and genitourinary tract. ${ }^{2}$ Involvement of the eye and nose is termed ophthalmomyiasis and nasal myiasis, respectively. Ophthalmomyiasis is further subdivided into internal and external types, dependent on the presence of intra-ocular penetration. ${ }^{3}$

Both nasal and ocular myiasis are rarely reported in the UK. We describe here a case of imported external ocular myiasis with nasal involvement presenting in Sunderland, northern England.

\section{Case report}

A 51-year-old woman presented to the accident and emergency department at Sunderland Royal Hospital, following referral by the ophthalmology team, complaining of a foreign body sensation in the right nasal cavity for the preceding two days.

She had initially described a seven-day history of feeling something in the right eye, after returning from a cruise in Morocco. The eye had become progressively itchy and congested, despite irrigation with topical antibiotics. A subsequent ophthalmology review (at Sunderland Eye Infirmary) had resulted in the removal of three larvae from the right eye under microscopic guidance; these larvae were sent for analysis.

Ten days after returning from Morocco, she had developed nasal symptoms and was referred to the ENT team for review.

On initial examination, no larvae were found within the nose, and the patient was treated conservatively in the community, with regular saline irrigation.
Four days later, the patient presented again with worsening nasal symptoms. On nasendoscopic examination, a single, photophobic larva was seen on the right inferior turbinate, and the right middle meatal region was congested and oedematous.

Discussion with the London School of Hygiene and Tropical Medicine confirmed that the initial larvae, retrieved by the ophthalmology team, were first stage larvae of Oestrus ovis, the sheep nasal bot fly.

We were advised to treat the patient with a single oral dose of the broad spectrum anti-parasitic drug invermectin $(200 \mu \mathrm{g} / \mathrm{kg})$ and removal of larvae.

Examination of both ears and the nasal cavity was performed under general anaesthesia. Two larvae were found, one still alive. Despite an extensive search, no other larvae were seen. Further findings included extremely congested nasal mucosa, despite treatment with phenylephrine, topical xylometazoline and adrenaline (1:10 000).

The patient was discharged the following day on regular xylometazoline nasal drops and antihistamines, with a follow-up appointment in seven days.

Subsequent review found no larvae and an improving nasal mucosa, despite the patient still experiencing nasal discomfort. Her decongestants and antihistamines were stopped. A review two days later found no resolution of symptoms, with worsening itching in the proximal nasal cavity. After further discussion with the London School of Hygiene and Tropical Medicine, a second dose of $12 \mathrm{mg}$ oral invermectin was administered.

On review a week later, the patient had mild rhinorrhoea and watering of the left eye. The nasal mucosa was mildly erythematous but there was no oedema. Once again, there was no sign of any larvae.

A final review, seven days later, revealed that all the patient's symptoms had subsided and her nasal mucosa appeared normal. She was discharged from further ENT follow up. 


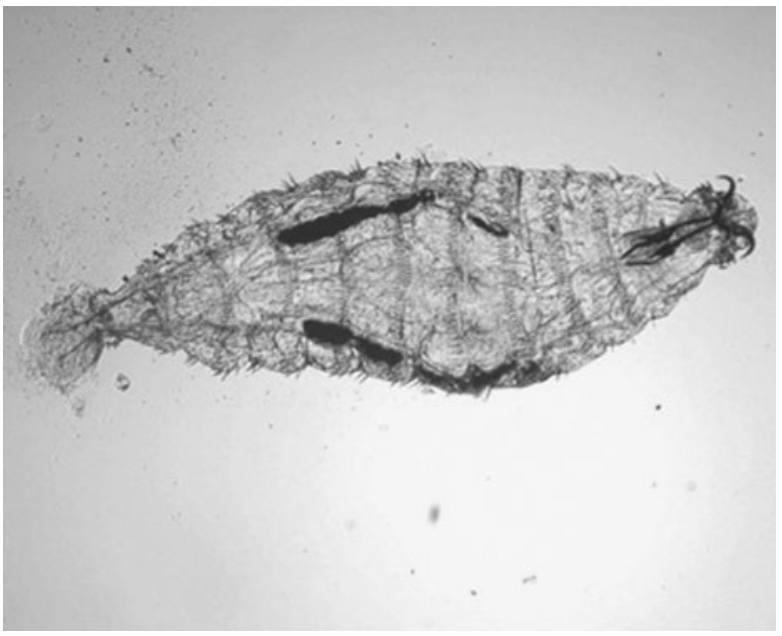

FIG. 1

A first instar Oestrus ovis collected from the eye of a patient in India presenting with conjunctivitis. Image courtesy of the $\mathrm{L} \mathrm{V}$ Prasad Eye Institute, Banjara Hills, Hyderabad, Andhra Pradesh, India.

\section{Discussion}

Oestrus ovis (sheep nasal bot fly) (class: Insecta; order: Diptera; family: Oestridae) is the most common cause of human myiasis. ${ }^{3,4}$ Oestrus ovis is generally regarded as benign and responsible for comparatively few ill effects. Sheep are the natural hosts; large, bee-like flies deposit their ova in the sheeps' nostrils. In their natural hosts, the larvae continue to develop, and migrate to the frontal sinuses. ${ }^{5}$ In the sinuses, the larvae mature through three progressively larger stages (instars) over a period of eight to 12 months (Figure 1). The fully matured larvae (third instar) pass out of the nostrils and pupate on the ground. Adult flies emerge from the pupae approximately three to six weeks later and live for approximately a month.

Occasionally, humans can act as incidental hosts when the larvae are injected near or onto the surface of the eyes or nasal mucosa. ${ }^{6}$ In humans, development of $O$ ovis larvae beyond the first instar stage is not seen, and it is believed they die within 10 days if not removed. ${ }^{3,6}$

- Myiasis is a parasitic disease caused by the larvae of numerous dipteran fly species, including the sheep bot fly Oestrus ovis

- This paper describes a rare case of a 51-year-old woman with ocular and nasal $\boldsymbol{O}$ ovis infestation

- Nasal myiasis is treated by removal of larvae, treatment with antiparasitic agents (under specialist advice) and nasal decongestants

Ocular myiasis is characterised by itching, burning, pain, redness and tearing in the affected eye. In extreme cases, the larvae may invade the globe of the eye, causing retinal damage and blindness. ${ }^{6}$ Penetration of the sinus mucosa causes pain, swelling and frontal headache. Nasal symptoms include foreign body sensation, itching, rhinorrhoea and sneezing. Cases of oral and pharyngeal myiasis have also been reported. ${ }^{7,8}$
Management of superficial $O$ ovis ocular infections involves removing the larvae under microscopic examination with the aid of topical anaesthesia to loosen larval attachments. ${ }^{8}$ Further treatment includes antibiotic and corticosteroid therapy to reduce the inflammatory response. Similarly, nasal myiasis is treated by removal of larvae, treatment with antiparasitic agents (under specialist advice) and nasal decongestants. Follow-up examination is advisable to exclude complications or the existence of additional larvae.

Oestrus ovis myiasis has a broad subtropical distribution, with the majority of reported cases occurring in the area from North Africa through to the Middle East and Southern Asia. ${ }^{2}$ Cases of myiasis within the UK, either indigenous or imported, remain rare. However, a history of recent travel to endemic areas should prompt a greater level of suspicion, and a detailed examination. Indeed, it is important for all healthcare providers to be aware of the diversity of disease which can present within the UK from an external source, as the potential risks from impromptu treatment can result in poor outcomes for our patients.

\section{Acknowledgements}

We thank Dr A W Berrington, Consultant Microbiologist, Sunderland Royal Hospital, and The London School of Hygiene and Tropical Medicine for their advice in managing this unusual case and assistance in identification of larvae.

\section{References}

1 Zumpt F, ed. Myiasis in Man and Animals in the Old World. A Textbook for Physicians, Veterinarians and Zoologists. London: Butterworths, 1965:175-9

2 Gregory A, Schatz S, Laubach H. Ophthalmomyiasis caused by the sheep bot fly Oestrus ovis in Northern Iraq. Optom Vis Sci 2004:81:586-90

3 Verstrynge K, Foets B. External ophthalmomyiasis: a case report. Bull Soc Belge Ophtalmol 2004;295:67-71

4 Chandra DB, Agrawal TN. Ocular myiasis caused by Oestrus ovis. Indian J Ophthalmol 1981;29:199-200

5 Badia L, Lund VJ. Vile bodies; an endoscopic approach to nasal myiasis. J Laryngol Otol 1994;108:1083-5

6 U.S Army Center for Health Promotion and Preventive Medicine. Entomological Sciences Program. In: http:// chppm-www.apgea.army.mil/ento [December 2008]

7 Hakimi R, Yazdi I. Oral mucosa myiasis caused by Oestrus ovis. Arch Iran Med 2002;5:194-6

8 Masoodi M, Hosseini K. The respiratory and allergic manifestations of human myiasis caused by larvae of the sheep bot fly (Oestrus ovis): a report of 33 pharyngeal cases from Southern Iran. Ann Trop Med Parasitol 2003; 97:75-81

Address for correspondence:

Miss Helen Cocks,

Consultant Otolaryngologist,

Sunderland Royal Hospital,

Kayll Road,

Sunderland SR4 7TP, UK.

E-mail: Helen.Cocks@chs.northy.nhs.uk

Miss $\mathrm{H}$ Cocks takes responsibility for the integrity of the content of the paper.

Competing interests: None declared 